Pacific Journal of Mathematics

APÉRY BASIS AND POLAR INVARIANTS OF PLANE CURVE 


\title{
APÉRY BASIS AND POLAR INVARIANTS OF PLANE CURVE SINGULARITIES
}

\author{
ANGEl Granja
}

Let $C$ be an irreducible plane algebroid curve singularity over an algebraically closed field $K$, defined by a power series $f \in K[[X, Y]]$. In this paper, we study those power series $h \in K[[X, Y]]$ for which the intersection multiplicity $(f \cdot h)=\operatorname{dim}_{K}(K[[X, Y]] /(f, y))$ is an element of the Apéry basis of the value semigroup for $C$. We prove a factorization theorem for these power series, obtaining strong properties of their irreducible factors. In particular we show that some results by $M$. Merle and $R$. Ephraim are a special case of this theorem.

Introduction. In this paper we denote by $K$ an algebraically closed field of arbitrary characteristic.

Let $C$ be an irreducible plane algebroid curve over $K$ (i.e. $C=$ $\operatorname{Spec}(R)$, where $R=K[[X, Y]] /(f)$, with $f$ irreducible). We will suppose $f \notin Y K[[X, Y]]$ and we will write $n=\operatorname{Ord}_{x}(f(X, 0))$.

We will denote by $S(C)$ the semigroup of values of $C$ (see [2], 11.0.1 and [3], 4.3.1), by $A_{n}=\left\{0=a_{0}<a_{1}<\cdots<a_{n-1}\right\}=$ $\left\{\min \left(S(C) n\left(k+n \mathbf{Z}_{+}\right) ; 0 \leq k \leq n-1\right\}\right.$ the Apéry basis of $S(C)$ relative to $n$ (see [2], 1.1.1) and by $\left\{v_{0}, \ldots, v_{r}\right\}$ the $n$-sequence in $S(C)$, where $v_{0}=n$, and $v_{i}=\min \left\{v \in S(C) ; \operatorname{gcd}\left(v_{0}, v_{1}, \ldots, v_{i-1}\right)>\right.$ $\left.\operatorname{gcd}\left(v_{0}, v_{1}, \ldots, v_{i-1}, v\right)\right\}, 1 \leq i \leq r($ see [1] $6.6,[2], 1.3 .2$ and [6]). $\left(\right.$ Note that $\operatorname{gcd}\left(v_{0}, \ldots, v_{r}\right)=1$.)

The main objective of this work is the proof of the following theorem.

Factorization Theorem. Let $h \in K[[X, Y]]$ be such that $0 \leq k=$ $\operatorname{Ord}_{x}(h(X, 0)) \leq n-1$. Then $(f \cdot h) \leq a_{k}$. Suppose $(f \cdot h)=a_{k}$. If $k=$ $\sum_{0 \leq q \leq r} s_{q}\left(n / d_{q-1}\right)$, where $d_{q}=\operatorname{gcd}\left(v_{0}, \ldots, v_{q}\right),\left(d_{0}=v_{0}=n, d_{r}=1\right)$, $0 \leq s_{q} \leq r$ and $0 \leq s_{q} \leq d_{q-1} / d_{q}$, then

$$
h=\prod_{1 \leq i \leq r} h_{i} \text { and } h_{i}=\prod_{1 \leq j \leq m_{i}} h_{i j}
$$

with $h_{i j}$ either irreducible or unit in $K[[X, Y]], 1 \leq j \leq m_{i}, 1 \leq i \leq r$, and

(1) $\sum_{1 \leq j \leq m_{i}} \operatorname{Ord}_{x}\left(h_{j}(X, 0)\right)=s_{i}\left(n / d_{i-1}\right), 1 \leq i \leq r$. 
(2) $\left(f \cdot h_{i j}(X, 0)\right)=d_{i-1} v_{i} / n$ if $s_{i} \neq 0$ and $h_{i j}$ is a unit in $K[[X, Y]]$ if $s_{i}=0,1 \leq j \leq m, 1 \leq i \leq r$.

Here $(f \cdot h)$ denotes, for two power series $f$ and $h$, the intersection multiplicity of the algebroid cycles defined, respectively, by $f$ and $h$.

In the fourth section we see that the polars of an irreducible complex analytic germ of a plane curve singularity satisfy the hypotheses of the above theorem for $k=n-1$. Thus, the Theorem 3.1 of [5] and Lemma 1.6 of [4] follow from the above Factorization Theorem.

1. Apéry basis and the $n$-sequence. In this section we will summarize some properties of the Apéry basis. For other properties you can see [2] and [6].

Proposition 1. If $M_{j}=K[[Y]]+K[[Y]] X+\cdots+K[[Y]] X^{j}, 0 \leq$ $j \leq n-1$, then:

(1) $\left\{a_{j}\right\}=v\left(M_{j-1}+X^{j}\right)-v\left(M_{j-1}\right), 1 \leq j \leq n-1$,

(2) $v\left(M_{j}\right)=\bigcup_{0 \leq i \leq j}\left(a_{i}+n \mathbf{Z}_{+}\right), 0 \leq j \leq n-1$,

(3) $a_{i}+a_{j} \leq a_{i+j}, 0 \leq i+j \leq n-1$,

where $v\left(M_{i}\right)=\left\{(f \cdot g) ; g \in M_{i}-\{0\}\right\}, 0 \leq i \leq n-1$ and $v\left(M_{i-1}+X^{i}\right)=$ $\left\{\left(f \cdot\left(g+X^{i}\right)\right) ; g \in M_{i-1}\right\}, 1 \leq i \leq n-1$.

Proof. See [2], Satz 3 and [6], Proposition 2.

REMARK 2. Note that in the above proposition $a_{j} \geq\left(f \cdot\left(g+X^{j}\right)\right)$ for each $g \in M_{j-1}, 1 \leq j \leq n-1$. (If $\left(f \cdot\left(g+X^{j}\right)\right)>a_{j}$, then there exists $g_{j-1} \in M_{j-1}$ such that $\left(f \cdot\left(g_{j-1}+X^{j}\right)\right)=a_{j}$, so $a_{j}=\left(f \cdot\left(g-g_{j-1}\right)\right)$ and we get a contradiction.)

Proposition 3. One has

$$
a_{s_{1}\left(d / d_{0}\right)+\cdots+s_{j}\left(d / d_{j-1}\right)}=s_{1} v_{1}+\cdots+s_{j} v_{j}
$$

and $v_{j+1}>\left(d_{j-1} / d_{j}\right) v_{j}, 0 \leq j \leq r-1$, with $0 \leq s_{i} \leq\left(d_{i-1} / d_{i}\right)$, $1 \leq i \leq r$.

Proof. See [2], Satz 2 and [6], Proposition 1.

REMARK 4. Note that $v_{j}=a_{d / d_{j}}, 1<j<r$ and

$$
A_{n}=\left\{a_{s_{1}\left(d / d_{0}\right)+\cdots+s_{r}\left(d / d_{r-1}\right)} ; 0 \leq s_{i}<\left(d_{i-1} / d_{i}\right), 1<i<r\right\} .
$$

EXAMPLE 5. Here we give some examples of different possibilities for the Apéry basis and $n$-sequences. Let us consider the curves 
$C_{i}=\operatorname{Spec}\left(K[[X, Y]] /\left(f_{i}\right)\right), 1 \leq i \leq 3$, where $f_{1}=X^{2}+Y^{5}, f_{2}=$ $\left(Y+X^{2}\right)^{2}+X^{5}$ and $f_{3}=Y^{2}+X^{5}$. It is easy to check that

$$
S\left(C_{1}\right)=S\left(C_{2}\right)=S\left(C_{3}\right)=\{0,2,4,5,6,7,8, \ldots\},
$$

and one has $f_{i} \notin Y K[[X, Y]], 1 \leq i \leq 3$, and $\operatorname{Ord}_{X}\left(f_{1}(X, 0)\right)=2$, $\operatorname{Ord}_{X}\left(f_{2}(X, 0)\right)=4$ and $\operatorname{Ord}_{X}\left(f_{3}(X, 0)\right)=5$. So $A_{2}=\left\{0=a_{0}\right.$, $\left.a_{1}=5\right\}$. The 2-sequence is $\left\{v_{0}=2, v_{1}=5\right\}, a_{1}=\left(f_{1} \cdot X\right)$, $d_{0}=d=2$ and $d_{1}=1 . A_{4}=\left\{0=a_{0}, a_{1}=2, a_{2}=5, a_{3}=7\right\}$. The 4-sequence is $\left\{v_{0}=4, v_{1}=2, v_{3}=5\right\}, a_{1}=\left(f_{2} \cdot X\right), a_{2}=$ $\left(f_{2} \cdot\left(Y+X^{2}\right)\right), a_{3}=\left(f_{2} \cdot\left(Y+X^{2}\right) X\right), d_{0}=d=4, d_{1}=2$ and $d_{2}=1$. And $A_{5}=\left\{0=a_{0}, a_{1}=2, a_{2}=4, a_{3}=6, a_{4}=8\right\}$. The 5 -sequence is $\left\{v_{0}=5, v_{1}=2\right\}, a_{i}=\left(f_{3} \cdot X^{i}\right), 1 \leq i \leq 4, d_{0}=d=5$ and $d_{1}=1$.

2. $n$-sequences and Hamburger-Noether expansions. Let $x$ and $y$ be, respectively, the residue classes of $X$ and $Y$ in $R$. Assume that $n_{0}=(f \cdot X) \leq(f \cdot Y)=n$, that is, $X$ is a generic coordinate (or $x$ is a transversal parameter of $C$, see [3]) and $Y$ could be generic, or have maximal contact with $f$, or any thing in between. In this form, we can study all of these possibilities for $Y$ simultaneously. This is the point of taking the Apéry basis with respect to a general $n$, rather than $n=n_{0}$. If $n=n_{0}$ then $Y$ should be generic.

Let

$$
\begin{aligned}
& y=a_{01} x+\cdots+a_{0 h_{0}} x^{h_{0}}+x^{h_{0}} z_{1}, \\
& x=z_{1}^{h_{1}} z_{2} \text {, } \\
& z_{s_{1}-1}=a_{s_{1} k_{1}} z_{s_{1}}^{k_{1}}+\cdots+a_{s_{1} h_{s_{1}}} z_{s_{1}}^{h_{s_{1}}}+z_{s_{1}}^{h_{S_{1}}} z_{S_{1}+1}, \\
& z_{s_{g}-1}=a_{s_{g} k_{g}} z_{s_{g}}^{k_{g}}+\cdots
\end{aligned}
$$

be the Hamburger-Noether expansion of $C$ in the basis $(x, y)$ (see [3], 2.2.2 and 3.3.4), and let $n_{i}=\operatorname{Ord}_{z_{s g}}\left(z_{i}\right), 0 \leq i \leq s_{g}\left(z_{0}=x\right)$, $\left(1=n_{s_{g}}<n_{s_{g}-1}<\cdots<n_{0} \leq n=\operatorname{Ord}_{z_{s g}}(y)\right.$, see [3], 2.2.5).

Note that the Hamburger-Noether expansion is nothing but an explicit description of the minimal resolution of singularities $\bar{C}$ of $C$ by a sequence of point blowing-ups. $z_{i}, z_{i-1}$ are the regular parameters of the ambient plane at the $h_{0}+\cdots+h_{i}$ th blowing up. $z_{s_{g}}$ is a regular parameter of $C$. In particular, for any $h \in K[[X, Y]]$ such that $f$ does not divide $h$

$$
(f \cdot h)=\operatorname{Ord}_{z_{s g}}(h) .
$$


The following proposition is an easy consequence of the HamburgerNoether expansion and the formula for Zariski exponents of a plane curve (see [3] 4.2.7 and 4.3.10).

Proposition 6. With the above notations one has:

(1) $n_{0}=\min (S(C)-\{0\})$,

(2) $n_{0} \leq n=v_{0} \leq h_{0} n_{0}+n_{1}$,

(3)(i) If $v_{0} \leq v_{1}$, then $r=g, v_{0}=n_{0}$ and

$$
v_{i+1}=\left(1 / n_{s_{i}}\right) \sum_{0 \leq j \leq s_{i}} h_{j} n_{j}^{2}+n_{s_{i}+1}
$$

$0 \leq i \leq r-1,\left(s_{0}=0\right)$. Moreover $a_{01} \neq 0$.

(ii) If $v_{0}>v_{1}$ and $d_{1}=v_{1}$, then $r=g+1, v_{0}=k_{0} v_{1}, k_{0} \geq 2, v_{1}=n_{0}$ and

$$
v_{i+2}=\left(1 / n_{s_{i}}\right) \sum_{0 \leq j \leq s_{i}} h_{j} n_{j}^{2}+n_{s_{i}+1}
$$

$0 \leq i \leq r-1,\left(s_{0}=0\right)$. Moreover $a_{0 j}=0,1 \leq j<k_{0}$ and $a_{1 k_{0}} \neq 0$.

(iii) If $v_{0}>v_{1}$ and $d_{1}<v_{1}$, then $r=g, v_{1}=n_{0}, v_{0}=h_{0} n_{0}+n_{1}$ and

$$
v_{i+1}=\left(1 / n_{s_{i}}\right) \sum_{0 \leq j \leq s_{i}} h_{j} n_{j}^{2}+n_{s_{i}+1}
$$

$0 \leq i \leq r-1,\left(s_{0}=0\right)$. Moreover $a_{0 j}=0,1 \leq j \leq h_{0}$.

Proof. (1) and (2) are obvious from the Hamburger-Noether expansions. We must only prove (3).

For this, if one writes $\bar{\beta}_{0}=n_{0}$ and

$$
\bar{\beta}_{i}=\left(1 / n_{s_{i}}\right) \sum_{0 \leq j \leq s_{i}} h_{j} n_{j}^{2}+n_{s_{i}+1},
$$

$0 \leq i \leq g-1$, then one has

(I) $\bar{\beta}_{0}=\min (S(C)-\{0\})$ and $\bar{\beta}_{i}=\min \left\{\bar{\beta} \in S(C) ; \operatorname{gcd}\left(\bar{\beta}_{0}, \ldots, \bar{\beta}_{i-1}\right)\right.$ $\left.>\operatorname{gcd}\left(\bar{\beta}_{0}, \ldots, \bar{\beta}_{i-1}, \bar{\beta}\right)\right\}, 1 \leq i \leq g$ (see [3], 4.2.7 and 4.3.10).

On the other hand, note that one has the equalities

(II) $v_{0}=n$ and $v_{i}=\min \left\{v \in S(C) ; \operatorname{gcd}\left(v_{0}, \cdots, v_{i-1}\right)>\right.$ $\left.\operatorname{gcd}\left(v_{0}, \ldots, v_{i-1}, v\right)\right\}, 1 \leq i \leq r$.

We distinguish the following three possibilities:

(i) $n_{0}=n<h_{0} n_{0}+n_{1}$. In that case $a_{01} \neq 0, v_{0}=n_{0}$ and it follows from (I) and (II) that $r=g$ and $v_{i}=\bar{\beta}_{i}, 1 \leq i \leq g$.

(ii) $n_{0}<n=k_{0} n_{0}<h_{0} n_{0}+n_{1}$. Then $a_{0 j}=0,1 \leq j \leq k_{0}, a_{0 k_{0}} \neq 0$, $v_{0}=k_{0} n_{0}, v_{1}=n_{0}$ and it follows from (I) and (II) that $r=g+1$ and $v_{i+1}=\bar{\beta}_{i}, 1 \leq i \leq r-1$. 
(iii) $n_{0}<n=h_{0} n_{0}+n_{1}$. Now $a_{0 j}=0,1 \leq j \leq h_{0}, v_{0}=h_{0} n_{0}+n_{1}$, $v_{1}=n_{0}$ and it follows from (I) and (II) that $r=g$ and $v_{i}=\bar{\beta}_{i}$, $2 \leq i \leq r$.

3. Infinitely near points and intersection multiplicity. Now consider another irreducible plane algebroid curve over $K, C^{\prime}=\operatorname{Spec}\left(R^{\prime}\right)$, with $R^{\prime}=K[[X, Y]] /\left(f^{\prime}\right), C^{\prime} \neq C$ and $f^{\prime} \notin Y K[[X, Y]]$. Let $x^{\prime}$ and $y^{\prime}$ be the residue classes of $X$ and $Y$, respectively, in $R^{\prime}$. We denote by

$$
\begin{aligned}
& y^{\prime}=a_{01}^{\prime} x^{\prime}+\cdots+a_{0 h_{0}^{\prime}}^{\prime} x^{h_{0}^{\prime}}+x^{h_{0}^{\prime}} z_{1}^{\prime}, \\
& x^{\prime}=z_{1}^{h_{1}^{\prime}} z_{2}^{\prime} \text {, } \\
& z_{s_{1}^{\prime}-1}^{\prime}=a_{s_{1}^{\prime} k_{1}^{\prime}}^{\prime} z^{k^{k_{1}^{\prime}}} s_{1}^{\prime}+\cdots+a_{s_{1}^{\prime} h_{s_{1}^{\prime}}^{\prime}}^{\prime} z_{s_{1}^{\prime}}^{h_{s_{1}^{\prime}}^{\prime}}+z_{s_{1}^{\prime}}^{h_{s_{1}^{\prime}}^{\prime}} z_{s_{1}^{\prime}+1}^{\prime} \text {, } \\
& z_{g_{g^{\prime}}-1}^{\prime}=a_{s_{g^{\prime}}^{\prime}, k_{g^{\prime}}^{\prime}}^{\prime} z_{s_{g^{\prime}}^{\prime}}^{k_{g^{\prime}}^{\prime}}+\cdots
\end{aligned}
$$

the Hamburger-Noether expansion of $C$ in the basis $\left(x^{\prime}, y^{\prime}\right)$. We also put $n_{i}^{\prime}=\operatorname{Ord}_{z_{s^{\prime}}^{\prime}}\left(z_{i}^{\prime}\right), 0 \leq i \leq s_{g^{\prime}}^{\prime},\left(x^{\prime}=z_{0}^{\prime}\right)$ and $n^{\prime}=\operatorname{Ord}_{x}\left(f^{\prime}(X, 0)\right)=$ $\operatorname{Ord}_{z_{s^{\prime}},}\left(y^{\prime}\right)$.

Let $N$ be the number of infinitely near points that $C$ and $C^{\prime}$ have in common (i.e. $N=h_{0}+h_{1}+\cdots+h_{s-1}+i-1, s$ being the largest integer for which $h_{q}=h_{q}^{\prime}, 0 \leq q \leq s-1$, and $a_{j k}=a_{j k}^{\prime}, i \leq k \leq h_{j}$, $0 \leq j \leq s-1$, and $i$ being the least index such that $a_{s i} \neq a_{s i}^{\prime}(i \leq$ $\left.\left.h_{s}+1, i \leq h_{s}^{\prime}+1\right)\right)($ see [3] 2.3.2).

Proposition 7. If

$$
\sum_{0 \leq q \leq s_{i-1}-1} h_{q}+k_{i-1}-1<N \leq \sum_{0 \leq q \leq s_{i}-1} h_{q}+k_{i}-1
$$

$1 \leq i \leq g,\left(s_{0}=0\right)$, then $\left(f \cdot f^{\prime}\right) \leq n^{\prime} d_{j-1} v_{j} / n$, where $j=i$ if $v_{0}<v_{1}$ or $v_{0}>v_{1}, d_{1}<v_{1}$, and $j=i+1$ if $v_{0}>v_{1}, d_{1}=v_{1}$. Furthermore, if $\left(f \cdot f^{\prime}\right)<n^{\prime} d_{j-1} v_{j} / n$, then $d_{j-1}$ divides $\left(f \cdot f^{\prime}\right)$.

Proof. One has $n=h_{q+1} n_{q+1}+n_{q+2}, s_{j} \leq q \leq s_{j+1}-2, n_{s_{j+1}-1}=$ $k_{j+1} n_{s_{j+1}}, 0<j \leq g-1$, and $n_{p}^{\prime}=h_{p+1}^{\prime} n_{p+1}^{\prime}+n_{p+2}^{\prime}, s_{j}^{\prime} \leq p \leq s_{j+1}^{\prime}-2$, $n_{s_{j+1}^{\prime}-1}^{\prime}=k_{j+1}^{\prime} n_{s_{j+1}^{\prime}}^{\prime}, 0<j \leq g^{\prime}-1$. 
So $n_{s_{i}}$ divides $n_{i}$, and $n_{s_{j}^{\prime}}^{\prime}$ divides $n_{k}^{\prime}$ for $i<s_{j}$ and $k<s_{j}^{\prime}$. On the other hand, since

$$
\sum_{0 \leq q \leq s_{i-1}-1} h_{q}+k_{i-1} \leq N
$$

then $h_{q}=h_{q}^{\prime}, 0 \leq q \leq s_{i-1}-1$ and $k_{i-1}=k_{i-1}^{\prime}$, so

(III) $n / n_{s_{i-1}}, n_{q} / n_{s_{i-1}}=n_{q}^{\prime} / n_{s_{i-1}}^{\prime}, 0 \leq q \leq s_{i-1}$.

From Proposition 5 we see that

(IV) $d_{j-1}=n_{s_{i-1}}$.

Thus, one can compute $\left(f \cdot f^{\prime}\right)$ in terms of the possible values of $N$ (see [3], 2.3.2 and 2.3.3). Namely, one has the following possibilities:

(A) $N=\sum_{0 \leq q \leq s_{i-1}-1} h_{q}+k_{i-1}$, with $k_{i-1}<k<\min \left(h_{s_{i-1}}, h_{s_{i-1}}^{\prime}\right)$.

In that case one has

$$
\begin{aligned}
\left(f \cdot f^{\prime}\right) & =\sum_{0 \leq q<s_{i-1}-1} h_{q} n_{q} n_{q}^{\prime}+k n_{s_{i-1}} n_{s_{i-1}}^{\prime} \\
& <\sum_{0 \leq q \leq s_{i-1}} h_{q} n_{q} n_{q}^{\prime}+n_{s_{i-1}+1} n_{s_{i-1}}^{\prime}=\alpha,
\end{aligned}
$$

so $d_{j-1}$ divides $\left(f \cdot f^{\prime}\right)$ by (IV), and $\alpha=n^{\prime} d_{j-1} v_{j} / n$, by (III), (IV) and Proposition 6.

(B) $N=\sum_{0 \leq q \leq s} h_{q}$, with $s_{i-1} \leq s<\min \left(s_{i}, s_{i}^{\prime}\right)$ and $h_{s}<h_{s}^{\prime}$.

Now one has

$$
\begin{aligned}
\left(f \cdot f^{\prime}\right) & =\sum_{0 \leq q \leq s} h_{q} n_{q} n_{q}^{\prime}+n_{s+1} n_{s}^{\prime} \\
& <\sum_{0 \leq q \leq s-1} h_{q} n_{q} n_{q}^{\prime}+h_{s}^{\prime} n_{s} n_{s}^{\prime}+n_{s} n_{s+1}^{\prime}=\beta .
\end{aligned}
$$

(Note that $h_{s}<h_{s}^{\prime}$, so $n_{s-1} n_{s}^{\prime}=h_{s} n_{s} n_{s}^{\prime}+n_{s+1} n_{s}^{\prime}<\left(h_{s}+1\right) n_{s} n_{s}^{\prime} \leq$ $h_{s}^{\prime} n_{s} n^{\prime}<h_{s}^{\prime} n_{s} n_{s}^{\prime}+n_{s} n_{s+1}^{\prime}$.) By (III), (IV) and Proposition 6, it follows that

$$
\begin{aligned}
& \left(f \cdot f^{\prime}\right)=\sum_{0 \leq q<s_{i-1}} h_{q} n_{q} n_{q}^{\prime}+n_{s_{i-1}+1} n_{s_{i-1}}=n^{\prime} d_{j-1} v_{j} / n, \text { or } \\
& \left(f \cdot f^{\prime}\right)=\sum_{0 \leq q<s_{i-1}} h_{q} n_{q} n_{q}^{\prime}+n_{s_{i-1}} n_{s_{i-1}+1}^{\prime}<\beta=n^{\prime} d_{j-1} v_{j} / n,
\end{aligned}
$$

and $d_{j-1}$ divides $\left(f \cdot f^{\prime}\right)$.

The other cases can be proved in a similar way:

(B') $N=\sum_{0 \leq q \leq s-1} h_{q}+h_{s}^{\prime}$, with $s_{i-1} \leq s<\min \left(s_{i}, s_{i}^{\prime}\right)$ and $h_{s}^{\prime}<h_{s}$.

(C.1) $N=\sum_{0 \leq q \leq s_{i}-1} h_{q}+k_{i}-1$, with $s_{i}<s_{i}^{\prime}$ and $k_{i}<h_{s_{i}}^{\prime}$.

(C.2) $N=\sum_{0 \leq q \leq s_{i}-1} h_{q}+h_{s_{i}}^{\prime}$, with $s_{i}<s_{i}^{\prime}$ and $h_{s_{i}}^{\prime}<k_{i}$. 
(C'.1) $N=\sum_{0 \leq q \leq s_{i}^{\prime}-1} h_{q}+k_{i}^{\prime}-1$, with $s_{i}^{\prime}<s_{i}$ and $k_{i}^{\prime}<h_{s_{i}^{\prime}}$.

(C'.2) $N=\sum_{0 \leq q \leq s_{i}^{\prime}-1} h_{q}+h_{s_{i}^{\prime}}$, with $s_{i}^{\prime}<s_{i}$ and $h_{s_{i}^{\prime}}^{\prime}<k_{i}^{\prime}$.

(D) $N=\sum_{0 \leq q<s_{i}-1} h_{q}+k_{i}-1$, with $s_{i}=s_{i}^{\prime}$ and $k_{i}<k_{i}^{\prime}$.

(D') $N=\sum_{0 \leq q \leq s_{i}-1} h_{q}+k_{i}-1$, with $s_{i}=s_{i}^{\prime}$ and $k_{i}^{\prime}<k_{i}$.

(E) $N=\sum_{0 \leq q<s_{i}-1} h_{q}+k_{i}-1$, with $s_{i}=s_{i}^{\prime}, k_{i}=k_{i}^{\prime}$ and $a_{s_{i} k_{i}} \neq a_{s_{i} k_{i}}^{\prime}$.

COROLlaRy 8. For each nonnegative integer $j, 1 \leq j \leq r$, the following statements are equivalent:

$$
\begin{gathered}
\left(f \cdot f^{\prime}\right)>n^{\prime} d_{j-1} v_{j} / n, \\
N=\sum_{0 \leq q<s_{i}-1} h_{q}+k_{i}-1,
\end{gathered}
$$

where $i=j$ if $v_{0}<v_{1}$ or $v_{0}>v_{1}$ and $d_{1}<v_{1}$, and $i=j-1, k_{0}=v_{0} / v_{1}$ if $v_{0}>v_{1}$ and $d_{1}=v_{1}$. In particular, if either (1) or (2) is true then $n^{\prime}=n_{s_{i}}^{\prime} n / d_{j}$.

Proof. (1) $\Rightarrow(2)$. If $v_{0}>v_{1}, d_{1}=v_{1}$ and $\left(f \cdot f^{\prime}\right)>n^{\prime} v_{1}$ then $N>k_{0}-1$. Indeed, suppose $N \leq k_{0}-1$. Then $a_{0 q}=a_{0 q}^{\prime}$, for $q \leq N$ and $a_{0 N+1} \neq a_{0 N+1}^{\prime}$. If $a_{0 N+1}^{\prime} \neq 0$ then $(N+1) n_{0}=n^{\prime}$ and if $a_{0 N+1}^{\prime}=0$ then $N+1=k_{0}$ and $(N+1) n_{0}^{\prime} \leq n^{\prime}$, so in any case $\left(f \cdot f^{\prime}\right)=(N+1) n_{0} n_{0}^{\prime} \leq n^{\prime} v_{1}$ and we get a contradiction.

Now suppose $\left(f \cdot f^{\prime}\right)>n^{\prime} d_{j-1} v_{j} / n$ and

$$
\sum_{0 \leq q \leq s_{i}-1} h_{q}+k_{i}-1<N
$$

with $j \geq 1$ if $v_{0}<v_{1}$ or $v_{0}>v_{1}$ and $d_{1}<v_{1}$, and with $j \geq 2$ if $v_{0}>v_{1}$ and $d_{1}=v_{1}$. Then we can assume

$$
\sum_{0 \leq q \leq s_{p-1}-1} h_{q}+k_{p-1}<N \leq \sum_{0 \leq q \leq s_{p-1}} h_{q}+k_{p}-1,
$$

with $1 \leq i \leq p$. It follows from Proposition 7 that $\left(f \cdot f^{\prime}\right) \leq n^{\prime} d_{s-1} v_{s} / n$, with $s \leq j$ and $d_{s-1} v_{s} \leq d_{j-1} v_{j}$ (see [2], Satz 2) which is a contradiction.

(2) $\Rightarrow$ (1). If $v_{0}>v_{1}, d_{1}=v_{1}$ and $N>k_{0}-1$, then $\left(f \cdot f^{\prime}\right)>k_{0} n_{0} n_{0}^{\prime}$, and $n^{\prime}=k_{0} n_{0}^{\prime},\left(a_{0 k_{0}}=a_{0 k_{0}}^{\prime}\right)$, so one has $\left(f \cdot f^{\prime}\right)>n^{\prime} v_{1}\left(n_{0}=v_{1}\right)$.

Now if

$$
\sum_{0 \leq q \leq s_{i}-1} h_{q}+k_{i}-1<N
$$


with $i \geq 1$ then $n / n_{s_{i}}=n^{\prime} / n_{s_{i}}^{\prime}, n_{q} / n_{s_{i}}=n_{q}^{\prime} / n_{s_{i}}^{\prime}, 0 \leq q \leq s_{i}$ and

$$
\left(f \cdot f^{\prime}\right)=\sum_{0 \leq q \leq s_{i}-1} h_{q} n_{q} n_{q}^{\prime}+k_{i} n_{s_{i}} n_{s_{i}}^{\prime}=\gamma .
$$

By Proposition 6

$$
\left(n^{\prime} / n\right) d_{j-1} v_{j}=\left(n_{s_{i-1}}^{\prime} / n_{s_{i-1}}\right)\left(\sum_{0 \leq q \leq s_{i-1}} h_{q} n_{q}^{2}+n_{s_{i-1}+1} n_{s_{i-1}}\right) .
$$

Now

$$
\gamma=\sum_{0 \leq q \leq s_{i-1}} h_{q} n_{q} n_{q}^{\prime}+k_{i} n_{s_{i}} n_{s_{i}}^{\prime}=\left(n_{s_{i-1}} / n_{s_{i-1}}\right)\left(\sum_{0 \leq q \leq s_{i-1}} h_{q} n_{q}^{2}+k_{i} n_{s_{i}}^{2}\right) \text {. }
$$

Thus we have to show that

$$
\sum_{0 \leq q \leq s_{i-1}} h_{q} n_{q}^{2}+n_{s_{i-1}+1} n_{s_{i-1}}=\sum_{0 \leq q \leq s_{i}-1} h_{q} n_{q}^{2}+k_{i} n_{s_{i}}^{2} .
$$

But this follows by repeated application of the identities $n_{q-1}=h_{q} n_{q}+$ $n_{q+1}$, since $k_{i} n_{s_{i}}=n_{s_{i}-1}$.

CoRollary 9. For $1 \leq j \leq r$, if $\left(f \cdot f^{\prime}\right)<n^{\prime} d_{j-1} v_{j} / n$, then $d_{j-1}$ divides $\left(f \cdot f^{\prime}\right)$.

Proof. If $v_{0}>v_{1}, d_{1}=v_{1}$ and $\left(f \cdot f^{\prime}\right)<n^{\prime} v_{1}$ then $N \leq k_{0}-1$ (Corollary 8). Thus, if $a_{0 q}=a_{0 q}^{\prime}, 1 \leq q \leq N$, and $a_{0 N+1} \neq a_{0 N+1}^{\prime}$ then $N+1=k_{0}$ and $\left(f \cdot f^{\prime}\right)=(N+1) n_{0} n_{0}^{\prime}=n_{0}^{\prime} v_{0}$. (For if $N+1<k_{0}$ then $\left(f \cdot f^{\prime}\right)=n^{\prime} v_{1}$ which is a contradiction.)

Now we can assume $\left(f \cdot f^{\prime}\right)<n^{\prime} d_{j-1} v_{j} / n$, with $j \geq 1$ if $v_{0}<v_{1}$ or $v_{0}>v_{1}$ and $d_{1}<v_{1}$, and $j \geq 2$ if $v_{0}>v_{1}$ and $d_{1}=v_{1}$. By Corollary 8 one has

$$
\sum_{0 \leq q \leq s_{i}-1} h_{q}+k_{i}-1 \geq N
$$

with $i=j$ if $v_{0}<v_{1}$ or $v_{0}>v_{1}$ and $d_{1}<v_{1}$, and with $i=j-1$ if $v_{0}>v_{1}$ and $d_{1}=v_{1}$. So, by Proposition 7, $d_{j-1}$ divides $\left(f \cdot f^{\prime}\right)$.

4. Proof of the Factorization Theorem. As $\operatorname{Ord}_{x}(h(X, 0))=k$ we can write $h=u h^{\prime}$, with $h^{\prime} \in M_{k-1}+X^{k}$ and $u \in K[[X, Y]]$ being a unit. So $(f \cdot h)=\left(f \cdot h^{\prime}\right) \leq a_{k}$.

Also, we can write $a_{k}=\sum_{0 \leq q \leq e} s_{q} v_{q}$ and $k=\sum_{0 \leq q \leq r} s_{q}\left(d / d_{q}\right)$, with $0 \leq s_{q}<d_{q-1} / d_{q}$ (see Remark 4). Let $q$ be the greatest index such that $s_{q} \neq 0$ and let

$$
h=\prod_{0 \leq j \leq m} h_{j}
$$


be the factorization of $h$ as a product of irreducible elements in $K[[X, Y]]$.

If for any $j$

$$
\left(f \cdot h_{j}\right) / \operatorname{Ord}_{x}\left(h_{j}(X, 0)\right)>d_{q-1} v_{q} / n
$$

then, by Corollary $8, \operatorname{Ord}_{x}\left(h_{j}(X, 0)\right)=a n / d_{q}(a \neq 0)$, but $k<n / d_{q}$ which is a contradiction. (Note that $s_{p}=0$ for $p>q$ and

$$
\left.k \leq \sum_{1 \leq p \leq q}\left(\left(d_{p-1} / d_{p}\right)-1\right)=\left(d / d_{q}\right)-1<d / d_{q}=n / d_{q} .\right)
$$

On the other hand, if for $1 \leq j \leq m$

$$
\left(f \cdot h_{j}\right) / \operatorname{Ord}_{x}\left(h_{j}(X, 0)\right)<d_{q-1} v_{q} / n
$$

then $d_{q-1}$ divides $(f \cdot h)$ by Corollary 9 . So $d_{q-1} / d_{q}$ divides $s_{q}$, and hence $s_{q}=0$ since $0 \leq s_{q}<d_{q-1} / d_{q}$, and we get a contradiction.

Thus, there exists $h_{j_{0}}$ such that

$$
\left(f \cdot h_{j_{0}}\right) / \operatorname{Ord}_{x}\left(h_{j_{0}}(X, 0)\right)=d_{q-1} v_{q} / n .
$$

Moreover, if $q \geq 2$ then $\operatorname{Ord}_{x}\left(h_{j_{0}}(X, 0)\right)=a n / d_{q-1}$ by Corollary 8, as $d_{q-1} v_{q}>d_{q} v_{q-1}$ (see Proposition 3). If $q=1$ then $\left(f \cdot h_{j_{0}}\right)=$ $\operatorname{Ord}_{x}\left(h_{j_{0}}(X, 0)\right)=a n / d_{q-1}$. In any case $\operatorname{Ord}_{x}\left(h_{j_{0}}(X, 0)\right)=a n / d_{q-1}$ with $0 \leq a \leq s_{q}$.

(Note that $k \leq \sum_{1 \leq p \leq q-1}\left(\left(d_{p-1}-1\right)-1\right)\left(d / d_{p-1}\right)+s_{q} d / d_{q-1}<$ $\left.\left(d / d_{q-1}\right)+s_{q} d / d_{q-1}=\left(s_{q}+1\right) d / d_{q-1}=\left(s_{q}+1\right) n / d_{q-1}.\right)$

So $h^{\prime}=h / h_{j_{0}}$ satisfies $\operatorname{Ord}_{x}\left(h^{\prime}(X, 0)\right)=k^{\prime}=k-a n / d_{q-1}$ and $\left(f \cdot h^{\prime}\right)=a_{k}-a\left(n / d_{q-1}\right) d_{q-1} v_{q} / n=a_{k}-a v_{q}=a_{k^{\prime}}$; hence the Theorem follows by iterating the above reasoning using $h^{\prime}$ instead of $h$ in the next step.

5. The complex analytic case. In this section, $C$ is assumed to be an irreducible complex analytic germ at $0 \in C^{2}$ of a plane curve singularity.

Let $n$ be the multiplicity of $C$ and let $P(C)$ be a general polar of $C$ (i.e. $P(C)$ is defined by a reduced element $h=\lambda(\partial f / \partial X)-\mu(\partial f / \partial Y)$ of $C\{X, Y\}$, and $n-1$ is the multiplicity of $P(C))$. M. Merle in [5] has proved that $P(C)$ descomposes into $g$ curves $\Gamma_{(1)}, \ldots, \Gamma_{(g)}$, where $\Gamma_{(g)}(1 \leq q \leq g)$ is such that

(1) its multiplicity is $\left(n / e_{q-1}\right)\left(\left(e_{q-1} / e_{q}\right)-1\right)$,

(2) every irreducible component of $\Gamma_{(q)}, \Gamma_{(q) i}$ has a contact of order $\beta_{q}$ with $C$ and $\left(\Gamma_{(q) i} \cdot C\right) / m\left(\Gamma_{(q) i}\right)=\bar{\beta}_{q} /(n / e)$. 
Here $\left\{\overline{\boldsymbol{\beta}}_{0}, \ldots, \overline{\boldsymbol{\beta}}_{g}\right\}$ is the minimal system of generators of $S(C), e_{q}=$ $\operatorname{gcd}\left(\bar{\beta}_{0}, \ldots, \bar{\beta}_{q}\right), 0 \leq q \leq g, \beta_{0}<\beta_{1}<\cdots<\beta_{g}$ are the Puiseux exponents and $m\left(\Gamma_{(q) i}\right)$ denotes the multiplicity of $\Gamma_{(q) i}$.

Without loss of generality, we may assume that $n=\operatorname{Ord}_{x}(f(X, 0))$, and therefore $n-1=\operatorname{Ord}_{x}(h(X, 0))$.

On the other hand,

$$
(f \cdot h)=\sum_{0 \leq q \leq g}\left(\left(e_{q-1} / e_{q}\right)-1\right) \bar{\beta}_{q} .
$$

and hence $(f \cdot h)=a_{n-1}$, since $\left\{\bar{\beta}_{0}, \ldots, \bar{\beta}_{g}\right\}$ is the $n$-sequence in $S(C)$ (see [2], Satz 2 and [5], Prop. 1.1).

Thus, $h$ satisfies the hypotheses of the Factorization Theorem for $k=n-1$, and the above Theorem 3.1 of [5] is a special case of ours. (Note that $\Gamma_{(q) i}$ has a contact of order $\beta_{q}$ with $C$ if and only if $\left(\Gamma_{(q) i} \cdot C\right) / m\left(\Gamma_{(q) i}\right)=\bar{\beta}_{q} /\left(n / e_{q-1}\right)$, see [5], Prop. 2.4.)

In general, if $M$ is a smooth germ of a plane curve singularity defined by $z \in C\{X, Y\}$, then the polar of $C$ with respect to $M$ is the (possibly nonreduced) germ whose defining ideal is generated by the Jacobian $J(f, z)=\partial(f, z) / \partial(X, Y)$ (see [4]). In particular, a general polar $P(C)$ of $C$ is defined by $h=J(f, \lambda X+\mu Y)$ with $(\lambda, \mu)$ general.

Thus, without loss of generality, we may assume that $z=Y$ (since $M$ is smooth) and $J(f, z)=\partial f / \partial X$.

Proposition 10. Keeping the above notations, one has

(a) $\operatorname{Ord}_{x}((\partial f / \partial X)(X, 0))=\operatorname{Ord}_{x}(f(X, 0))-1=n-1$.

(b) $(f(\partial f / \partial X))=a_{n-1}$.

Proof. (a) It is obvious.

(b) If $n=\operatorname{Ord}_{x}(f(X, 0)) \geq \operatorname{Ord}_{Y}(f(0, y))=m$ then one has a Puiseux type parametrization of $C$

$$
X=t^{m}, \quad Y=\Psi(t)
$$

and we can write (up to multiplication by a unit)

$$
\left.f(X, Y)=\prod_{0 \leq q \leq m}\left(X-\Psi\left(W^{q} X^{1 / m}\right)\right)\right)
$$

Thus,

$$
\begin{aligned}
(f \cdot(\partial f / \partial X)) & =\operatorname{Ord}_{t}\left((\partial f / \partial X)\left(t^{m}, \Psi(t)\right)\right) \\
& =\operatorname{Ord}_{t}\left(\Psi^{1}\left(t^{m}\right)\right)+\operatorname{Ord}_{t}\left(\prod_{1 \leq q \leq m-1}\left(\Psi(t)-\Psi\left(W^{q} t\right)\right) .\right.
\end{aligned}
$$

where $\Psi^{1}\left(X^{1 / m}\right)=\partial / \partial X\left(\Psi\left(X^{1 / m}\right)\right)$. 
On the other hand, we can write

$$
\begin{aligned}
\Psi\left(X^{1 / m}\right)= & \sum_{1 \leq j \leq i_{0}} a_{0 j} X^{j n / m} \\
& +\sum_{0 \leq j \leq i_{1}} a_{1 j} X^{\left(\beta_{1}+j e_{1}\right) / m}+\cdots+\sum_{0 \leq j} a_{g j} X^{\left(\beta_{g}+j e_{g}\right) / m},
\end{aligned}
$$

where $m=\beta_{0}<\beta_{1}<\cdots<\beta_{g}$ are the Puiseux exponents of $C$ and $e_{i}=\operatorname{gcd}\left(\beta_{0}, \ldots, \beta_{i}\right), 1 \leq i \leq g$.

Then we have $\operatorname{Ord}_{t} \Psi^{1}\left(X^{1 / n}\right)=n-m$, and

$$
\operatorname{Ord}\left(\prod_{1 \leq q \leq m-1}\left(\Psi(t)-\Psi\left(w^{q} t\right)\right)\right)=\sum_{1 \leq q \leq g}\left(e_{i-1}-e_{i}\right) \beta_{i}
$$

(Note that $\operatorname{Ord}_{t}\left(\Psi(t)-\Psi\left(w^{q} t\right)\right)=\beta_{j}$, if

$$
\begin{aligned}
q \in\left\{k\left(e_{j-2} / e_{j-1}\right) ; 1 \leq k<e_{j-1}\right\}-\left\{k\left(e_{j-1} / e_{j}\right)\right. & \left.; \leq k<e_{j}\right\}, \\
1 & \left.\leq j \leq g \quad\left(e_{-1}=e_{0}=m\right) .\right)
\end{aligned}
$$

Now

$$
\sum_{1 \leq i \leq g}\left(e_{i-1}-e_{i}\right) \beta_{i}=c+m-1,
$$

where $c$ is the conductor of $S(C)$ (i.e. $c=\min \left\{d \in S(C) ; d+\mathbf{Z}_{+} \subset\right.$ $S(C)\}$, see [3], 4.4) and $c+n-1=a_{n-1}$, since

$$
A_{n}=\left\{\min \left(S(C) \cap\left(j+n \mathbf{Z}_{+}\right) ; 0 \leq j \leq n-1\right\} .\right.
$$

Finally, a similar argument shows that $(f \cdot \partial f / \partial X)=c+n-1$, if $n=\operatorname{Ord}_{x}(f(X, 0))<\operatorname{Ord}_{Y}(f(0, Y))$.

Remark 11. Proposition 10 shows that if $h$ defines the polar of $C$ with respect to $M$ then $h$ satisfies the hypotheses in the Factorization Theorem for $k=n-1$, so Lemma 1.6 of [4] is also a special case of (2) in the Factorization Theorem.

Acknowledgments. I would like to the thank the referee for this valuable suggestions and kind help.

\section{REFERENCES}

[1] S. S. Abhyankar, Expansion Techniques in Algebraic Geometry, Tata Institute of Fundamental Research, Bombay, (1977).

[2] G. Angermuller, Die Wertehalgruppe einer ebenen irreduziblen algebroiden Kurve, Math. Z., 153 (1977), 267-282. 
[3] A. Campillo, Algebroid Curves in Positive Characteristic, Lecture Note No. 813, Springer-Verlag, (1980).

[4] R. Ephraim, Special polars and curves with one place at infinity, Proc. Symp. Pure Math., 40, Part 1 (1983).

[5] M. Merle, Invariant polaires des courbes planes, Invent. Math., 41 (1977), 103111.

[6] H. Pinkham, Courbes planes ayant une seule place a l'infini, Publications de Centre de Mathematiques. Ecole Polytechnique, F. 91128 Palaiseu, (19771978).

[7] B. Teissier, Varietes polaires I. Invariants polaires des singularities des hypersurfaces, Invent. Math., 40 (1977), 267-298.

[8] O. Zariski, Modules de branches planes, Publications de Centre de Mathematiques. Ecole Polytechnique, F. 91128, Palaiseau (1973).

Received December 31, 1986 and in revised form November 18, 1988.

Facultad de Ciencias

UNIVERSIDAD DE VALLADOLID

47005-VAlladolid, SPain

AND

FACULTAD DE VETERINARIA

UNIVERSIDAD DE LEON

24007-Leon, SPain 


\section{PACIFIC JOURNAL OF MATHEMATICS EDITORS}

\author{
V. S. VARADARAJAN \\ (Managing Editor) \\ University of California \\ Los Angeles, CA 90024-1555-05 \\ Herbert Clemens \\ University of Utah \\ Salt Lake City, UT 84112 \\ THOMAS ENRIGHT \\ University of California, San Diego \\ La Jolla, CA 92093
}

R. FINN

Stanford University

Stanford, CA 94305

HeRMANN FlaschKa

University of Arizona

Tucson, AZ 85721

VAUGHaN F. R. Jones

University of California

Berkeley, CA 94720

STEVEN KeRCKHOFF

Stanford University

Stanford, CA 94305

\author{
ROBION KIRBY \\ University of California \\ Berkeley, CA 94720 \\ C. C. MOORE \\ University of California \\ Berkeley, CA 94720 \\ HAROLD STARK \\ University of California, San Diego \\ La Jolla, CA 92093
}

\section{ASSOCIATE EDITORS}
R. ARENS
E. F. BECKENBACH
B. H. NEUMANN
F. WOLF
K. YoshidA (1906-1982)

\section{SUPPORTING INSTITUTIONS}

UNIVERSITY OF ARIZONA

UNIVERSITY OF BRITISH COLUMBIA

CALIFORNIA INSTITUTE OF TECHNOLOGY

UNIVERSITY OF CALIFORNIA

MONTANA STATE UNIVERSITY

UNIVERSITY OF NEVADA, RENO

NEW MEXICO STATE UNIVERSITY

OREGON STATE UNIVERSITY
UNIVERSITY OF OREGON

UNIVERSITY OF SOUTHERN CALIFORNIA

STANFORD UNIVERSITY

UNIVERSITY OF HAWAII

UNIVERSITY OF TOKYO

UNIVERSITY OF UTAH

WASHINGTON STATE UNIVERSITY

UNIVERSITY OF WASHINGTON 


\section{Pacific Journal of Mathematics}

Vol. 140, No. $1 \quad$ September, 1989

Michel Brestovski, Algebraic independence of solutions of differential

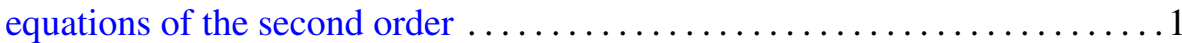

Bohumil Cenkl, Cohomology operations from higher products in the de

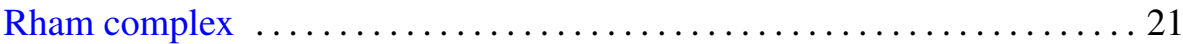

Gustavo Corach and Daniel Suarez, Generalized rational convexity in

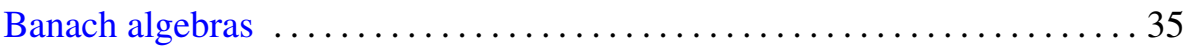

Keresztély Corrádi and Sándor Szabó, A new proof of Rédei’s theorem . . . 53

Steven R. Costenoble and Stefan Waner, Equivariant orientations and

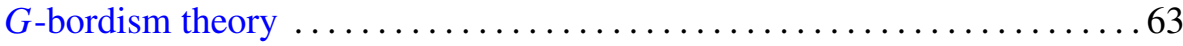

Angel Granja, Apéry basis and polar invariants of plane curve

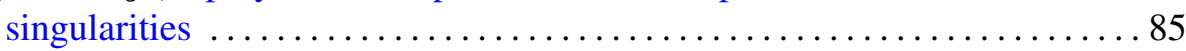

Young Soo Jo, Isometries of tridiagonal algebras .................. 97

Ronald Leslie Lipsman, Harmonic analysis on exponential solvable homogeneous spaces: the algebraic or symmetric cases $\ldots \ldots \ldots \ldots \ldots 117$

Erich Miersemann, On the behaviour of capillaries at a corner 149

Marian Nowak, On the finest Lebesgue topology on the space of essentially

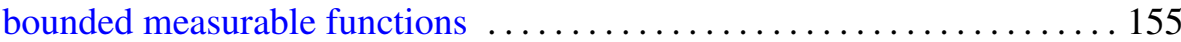

Pascal J. Thomas, Hardy interpolating sequences of hyperplanes ........ 163

H. Bevan Thompson, Differentiability properties of subfunctions for second order ordinary differential equations 